\title{
CARACTERÍSTICAS DE LA MUJER JUGADORA PATOLÓGICA (1)
}

\author{
Elisardo Becoña Iglesias (2) \\ Universidad de Santiago de Compostela
}

\begin{abstract}
RESUMEN
Con el surgimiento de los primeros casos de jugadores patológicos en España se ha apreciado, de modo semejante a otros paises, que hay caracteristicas diferenciadoras entre hombres y mujeres jugadoras patológicos. Después de analizar la prevalencia de este trastorno en las mujeres en España se presentan doce características que permiten delimitar los rasgos más relevantes en la mujer jugadora patológica: adquisición del trastorno, tipo de juego, la doble moral social ante el juego de la mujer, fases por las que pasa su juego, problemas de depresión, la reacción de su marido y el sufrimiento de sus hijos, su mayor control en la obtención de dinero para jugar, su comienzo más tarde en el juego, su mayor nivel de psicopatologia, sus antecedentes familiares (juego, alcohol) y el tratamiento.
\end{abstract}

Palabras clave: Juego patológico, psicopatología y mujer, mujer jugadora patológica.

\begin{abstract}
In Spain, as in other countries, with the appearance of pathological gambleing differentiating characteristics have been observed between men and women pathological gamblers. After analyzing the prevalence of this disorder among women in Spain twelve characteristics show up which allow us to isolate the most relevant traits of the women pathological gambler: development of the disorder, type of game, double morality in the society concerning the gambling of women, the stages of gambling, problems of depression, the reaction of the husband and the suffering of the children, her greater control in obtaining money for gambling, the later initiation into gambling, her greater degree of psychopathology, family antecedents (gambling, alcohol) and the treatment.
\end{abstract}

Key words: Pathological gambling, woman and psychopathology, woman pathological gambler.

\footnotetext{
1 Una primera versión de este artículo fue presentada como Conferencia Inaugural del VI Congreso Andaluz de Jugadores de Azar Rehabilitados FAJER, Diciembre de 1996, en Granada.

${ }^{2}$ Correspondencia: Elisardo Becona Iglesias. Universidad de Santiago de Compostela. Facultad de Psicologla. Departamento de Psicologia Clinica y Psicobiologia. Campus Universitario Sur. 15706 Santiago de Compostela.
} 


\section{INTRODUCCIÓN}

El juego por ocio o placer es una actividad normal en la vida de cualquier niño y en la de los adultos. Pero hay un juego, el de azar, que acarrea graves problemas en un porcentaje significativo de personas. Por él entendemos a aquel juego en el cual nosotros no podemos poner en marcha ninguna habilidad para controlar el resultado del mismo. Juegos como las máquinas tragaperras, el bingo, las loterias, son juegos de azar. En ellos no tenemos control sobre el mismo ni sobre su resultado. Depende del azar, de un procedimiento mecánico que selecciona al azar un número, sin que podamos hacer nada porque salga uno $\mathrm{u}$ otro $\mathrm{ni}$ podemos saber cual va a salir en vez de otro.

Consideramos, por tanto, que una persona tiene problemas de juego, ludopatía o juego patológico, cuando su juego le acarrea graves problemas tanto a nivel personal, como familiar, profesional y social. Piensa, vive y actúa en función del juego (American Psychiatric Association, 1994; OMS, 1992). Su vida la organiza en torno al juego, dejando a un lado cualquier otro tipo de objetivo que no sea el juego. Por tanto, es un esclavo del juego (Becoña, 1996).

Cuando juega obtiene un gran placer. Cuando no puede jugar se encuentra mal y procura por todos los medios volver a jugar lo antes posible. Las pérdidas le fuerzan a jugar, cada vez mayores cantidades de dinero, para recuperar lo que ha perdido, mediante el juego. Al menos eso es lo que piensa. Entra asi en un círculo vicioso del que es dificil salir.

El juego de azar no es un fenómeno de hoy. Jugar por placer, ocio o por dinero, tiene muchos miles de años de antigüedad y ha estado presente en casi todas las culturas (Becoña, 1996). Los babilónicos, etruscos, egipcios, chinos, indios precolombinos, griegos, romanos, etc. tenian distintos juegos de azar (Fleming, 1978). Sin embargo el boom reciente del juego de azar se produce hace pocos años, a partir de 1977, cuando se legaliza formalmente el juego de azar en toda su extensión. En pocos años, a partir de esta fecha, se fueron legalizando otros juegos de azar, como el casino, el bingo, las máquinas tragaperras, nuevas loterias, hasta llegar a un gran número de juegos en la actualidad (González, 1989).
El motivo económico ha estado en la base de este nuevo fenómeno. El juego es muy rentable tanto para los operadores como para la administración recaudatoria. También produce muchos puestos de trabajo y es una de las actividades más importantes de la economía de muchos paises. Hablar de un gasto en juego, como el que está ocurriendo en España, de más de 3 billones de pesetas al año es una prueba evidente. De esta enorme cantidad de dinero las máquinas tragaperras representan más de un billón de pesetas, en el bingo 630.000 millones, en las loterias 750.000 millones y en el cupón de la ONCE casi 370.000 millones de pesetas. Los otros juegos tienen cantidades menores, y los casinos 155.000 millones (Comisión Nacional del Juego, 1996).

Pero el quid de la cuestión del juego patológico en España ha sido la legalización y enorme expansión de las máquinas tragaperras (Ochoa y Labrador, 1994). Por su poder adictivo, por la inmediatez del premio y por su disponibilidad pronto se convirtieron en muy populares, a partir de su legalización en 1981. A diferencia de casi todos los países, en España, se permitió que éstas se colocasen no solo en casinos y locales preparados al efecto, sino en bares, restaurantes, lugares de diversión, etc. Esto lo vemos hoy reflejado en los jugadores existentes.

Pero hablando de la mujer jugadora, que es nuestro objetivo, son otros dos juegos los que más la enganchan: el bingo y los cupones y loterias.

El bingo surgió con gran fuerza una vez legalizado, en 1977. Era algo nuevo que habia que probar. Pronto surgieron bingos en todas partes. Pronto las parejas y no parejas iban a jugar al bingo. También las amigas, los conocidos, pasaban un rato en él. Algunas de éstas quedaron enganchadas en él. Algo semejante ha pasado y pasa con las mujeres enganchadas a las loterias, que ellas creen que compran por un fin caritativo $y$, a una parte de ellas, les está destrozando la vida.

\section{LOS DATOS ACTUALES SOBRE EL JUEGO DE LAS MUJERES EN ESPAÑA}

Hoy tenemos estudios lo suficientemente fiables como para conocer los datos sobre el número de jugadores patológicos que hay en España. 
El incremento en la oferta de juegos, la expansión del número de jugadores $\mathrm{y}$, consiguientemente, de jugadores patológicos, en los países de nuestro entorno o de otros continentes, ha sido semejante al nuestro en los últimos años. En los próximos, es previsible que la oferta de juegos de azar se mantenga en los actuales niveles o, más probablemente, se incremente.

Por los estudios parciales que se han realizado en Galicia (Becoña, 1993; Becoña y Fuentes, 1995), Cataluña (Cayuela, 1990) y Andalucia (Legarda, Babio y Abreu, 1992; Irurita, 1996), podemos tener una idea clara de la situación del juego patológico en estas comunidades y poder extrapolar con un relativo margen de seguridad al resto del estado el número de jugadores patológicos. En esta linea, nuestra estimación (Becoña, Labrador, Echeburúa, Ochoa y Vallejo, 1995) es que existen no menos de un $1.5 \%$ de jugadores patológicos adultos y un $2 \%$ de jugadores problemas, o personas que pueden tener problemas de adicción en el futuro. Esto en cifras significa que existen aproximadamente 450.000 jugadores patológicos adultos y 750.000 jugadores problema.

Tabla 1.- Características de los jugadores patológicos en Galicia en distintas variables demográficas

\begin{tabular}{|c|c|c|}
\hline & DSM-III-R (1) & SOGS (2) \\
\hline \multicolumn{3}{|l|}{ Sexo (\%) } \\
\hline Hombre & 67.9 & 63.6 \\
\hline Mujer & 32.1 & 36.4 \\
\hline \multicolumn{3}{|l|}{ Edad (\%) } \\
\hline $18-30$ & 39.2 & 36.4 \\
\hline $31-45$ & 28.6 & 36.4 \\
\hline $46-64$ & 21.4 & 27.3 \\
\hline$>64$ & 10.7 & 0.0 \\
\hline \multicolumn{3}{|c|}{ Estado civil (\%) } \\
\hline Soltero & 35.7 & 36.4 \\
\hline Casado & 53.6 & 63.6 \\
\hline Viudo & 7.1 & 0.0 \\
\hline $\begin{array}{l}\text { Separado/ } \\
\text { divorciado }\end{array}$ & 3.6 & 0.0 \\
\hline \multicolumn{3}{|c|}{$\begin{array}{l}\text { (1) } N=1.615, \text { representativa de las ciudades de Galicia } \\
\text { (2) } N=1.028, \text { representativa de toda Galicia }\end{array}$} \\
\hline \multicolumn{3}{|c|}{ Fuente: Becoña (1993) y Becona y Fuentes (1995). } \\
\hline
\end{tabular}

Tabla 2.- Características de los jugadores patológicos en Andalucía en distintas variables demográficas

\begin{tabular}{|c|c|c|}
\hline & $\begin{array}{l}\text { Jugadores } \\
\text { adictos }\end{array}$ & $\begin{array}{l}\text { Jugadores } \\
\text { dependientes }\end{array}$ \\
\hline \multicolumn{3}{|l|}{ Sexo (\%) } \\
\hline Hombre & 82.0 & 93.3 \\
\hline Mujer & 18.0 & 6.7 \\
\hline \multicolumn{3}{|l|}{ Edad (\%) } \\
\hline $18-30$ & 31.3 & 33.7 \\
\hline $31-43$ & 24.2 & 22.5 \\
\hline $44-56$ & 18.5 & 30.3 \\
\hline$>56$ & 26.1 & 13.5 \\
\hline \multicolumn{3}{|c|}{ Estado civil (\%) } \\
\hline Soltero & 37.1 & 39.3 \\
\hline Casado & 53.8 & 53.9 \\
\hline Viudo & 7.1 & 1.1 \\
\hline $\begin{array}{l}\text { Separado/ } \\
\text { divorciado }\end{array}$ & 1.9 & 5.6 \\
\hline \multicolumn{3}{|c|}{$\begin{array}{l}\text { Jugadores adictos = probable jugador problema } \\
\text { Jugador dependiente= probable jugador patológico }\end{array}$} \\
\hline \multicolumn{3}{|c|}{ Fuente: Inurita (1996). } \\
\hline
\end{tabular}

Respecto a las mujeres, los datos existentes indican que éstas representan del total de jugadores patológicos un amplio rango de una comunidad autónoma a otra. Siguiendo los estudios más amplios y fiables existentes, tendriamos que el porcentaje de mujeres jugadores patológicas oscilaría del $7 \%$ en Andalucia al $36 \%$ en Galicia del total de los jugadores patológicos existentes en esas comunidades (ver tablas 1 y 2). Del total de jugadores que acuden a tratamiento, las mujeres suelen representar el $10 \%$, tanto en las asociaciones de Jugadores Anónimos de otros países como en las Asociaciones de Jugadores en Rehabilitación en España; en algunos programas clínicos en España esta relación puede subir hasta el $20 \%$ de mujeres (ej., Echeburúa, Báez y Fernández-Montalvo, 1996; Saiz, Moreno y López-lbor, 1992)

Hay claras diferencias en cuanto al tipo de juego en uno y otro sexo. Como señalan muchos autores, los hombres juegan más a los juegos activos e inmediatos (ej. máquinas tragaperras) y las mujeres a los pasivos (ej. loterias y cupones), aunque hay una excepción en el juego del bingo, donde acuden a ellos 
por igual hombres que mujeres y en algunas ocasiones y horas más mujeres que hombres. Un juego especial para la mujer lo constituyen los cupones y loterias. Vienen a ser la "piel de oveja" de un problema muy grave para muchas personas, especialmente el cupón de la ONCE, que resulta ser muy adictivo y en donde los jugadores "subjetivamente" creen que no se pueden estar haciendo nada malo a ellos mismos dado que colaboran en un "fin benéfico". Este fin benéfico, a algunos, les va a representar su ruina personal y familiar.

\section{LA MUJER JUGADORA PATOLÓGICA}

Como ocurre con la mujer dependiente del alcohol (Edwards, 1986), hay importantes paralelismos en las características de la mujer jugadora patológica, especialmente en la diferencia por sexo. Destaca especialmente, como ampliaremos posteriormente, una valoración social más negativa de su adicción y un menor apoyo en su medio más cercano (ej. familiar) y de la sociedad en general, que el que tiene el varón.

El papel tradicional de la mujer, o su papel moderno como mujer, que implica una carga de trabajo aún mayor, va a incidir en su recuperación, dependiendo del tipo de juego, edad, estado civil, tener o no hijos, estado emocional y psicopatologia asociada, entre los más relevantes.

Por suerte, y siguiendo la línea de comparación con el alcohol, por su relevancia en nuestro medio, dado el mayor problema de metabolización biológico del alcohol por ser mujer, lo que facilita que haga un uso moderado del mismo o no lo consuma, la menor asistencia en relación con los hombres a bares y lugares de bebida, la asunción social de que una mujer en un bar no es su sitio "natural", favorece que esta variable, el consumo de aicohol (Echeburúa, 1996), que tiene en España una gran relevancia en el juego más adictivo, las máquinas tragaperras, sea un factor de protección en la mujer respecto al juego patológico. Pero ello no debe hacernos olvidar que el juego patológico también existe en la mujer, y existe en otros juegos, juegos que le son más adaptativos a su vida cotidiana y que en ocasiones pasan desapercibidos que causan problemas, también graves problemas en muchas mujeres.
Hoy todos los autores más relevantes en el campo del juego patológico reconocen la poca información que existe sobre la mujer jugadora patológica (ej., Custer y Milt, 1985; Lesieur, 1988, 1993a; Heineman, 1992), a diferencia de lo que ocurre en los hombres. Como afirmaban Custer y Milt (1985) hace diez años "sólo podemos dar impresiones, no conclusiones" (p. 146). Hoy conocemos un poco más del tema, pero hay que reconocer que todavia queda un largo camino por recorrer para conocer mejor la situación de la mujer jugadora patológica $y$, lo que es más importante, que acuda a los programas de tratamiento y no quede en su casa sin buscar ayuda.

En las páginas que siguen presentamos los datos disponibles para hacer una radiografia de la situación de la mujer jugadora patológica, tanto siguiendo los datos que existen sobre el juego en general, y que son aplicables por igual a ambos sexos, como algunas características que diferencian claramente a la mujer jugadora patológica respecto al varón con el mismo problema. Estos datos puede agruparse en 12 características:

1. La mujer jugadora patológica poco o nada se diferencia del varón en los procesos psicológicos que llevan a la adquisición de la conducta de juego, a la escalada hacia un juego problemático y posterior aparición del juego patológico.

Son varias las razones que explican el que los juegos de azar produzcan en España tan graves problemas de dependencia. A pesar de que se han propuesto varias (ej., Becoña, 1993, 1995a, 1995b, 1996; Becoña et al., 1995; Brown, 1988; Labrador y Becoña, 1994; Ochoa y Labrador, 1995; Robert y Botella, 1995; Walker, 1992), hay acuerdo que los factores que se relacionan con el desarrollo y aparición de este problema son los siguientes: la gran disponibilidad de juegos; la gran accesibilidad al juego; el bajo coste del juego; la promoción y publicidad de algunos juegos de azar; la interacción de otras sustancias adictivas con el juego; los factores de vulnerabilidad al juego; $y$, el gran poder adictivo de ciertos juegos de azar

Lo anterior, expuesto de un modo lineal, nos permite explicar el proceso por el que una persona pasa desde su primer contacto con el juego hasta convertirse en un jugador patolo- 
gico (Becoña, 1995a, 1996; Labrador y Becoña, 1994):

1) La exposición al juego, y por tanto la disponibilidad de juegos de azar, va a facilitar el que se de inicialmente esta conducta y que con posterioridad pueda ser abusiva.

2) La existencia de ciertos factores de predisposición en las personas que parecen favorecer la aparición del juego excesivo.

Se han apuntado varios factores de predisposición para el juego patológico. Estos favorecen su aparición. Entre ellos se han apuntado el nivel de activación fisiológico, especialmente el nivel de activación o arousal, factores de personalidad (ej., búsqueda de sensaciones y aventuras), educación familiar y valores sociales y culturales sobre el juego, facilidad de acceso al juego, creencias y pensamientos irracionales sobre el juego, eventos vitales negativos a los que se busca refugio en el juego.

3) Una vez que se han comenzado a desarrollar conductas de juego éstas se mantendrán o no en función de las consecuencias percibidas (reforzamiento positivo y negativo) junto al siempre presente programa de reforzamiento de razón variable.

4) La exposición al juego, los factores de predisposición, las consecuencias del juego, creencias y pensamientos irracionales y los eventos vitales negativos pueden conducir a la persona a un tipo de juego problemático o excesivo, que puede ser temporal o hacerse más intenso y crónico y desembocar en el juego patológico.

5) La falta de habilidades adecuadas para hacer frente a la conducta de juego e impedir su mantenimiento y desarrollo, en especial si existe un cierta disponibilidad económica (propia u obtenida por medios legales o ilegales), facilitará la cronificación del juego excesivo. El jugador irá pasando por las fases de ganancia, pérdida y desesperación.

6) Cuando llega a esta fase final lo más probable es que el jugador patológico tenga ya serios problemas de todo tipo: personales, familiares, laborales, sociales, financieros $y$ legales. En estos momentos algunos pedirán ayuda, otros tratarán de solucionar autónomamente su situación, otros continuarán con estas conductas hasta su total ruina personal, pudiendo llegar incluso al suicidio.
7) La salida de esta situación puede ser dificil incluso con la ayuda de profesionales expertos, siendo determinante la adquisición de distintas habilidades (personales, relacionales, comunicativas, tiempo libre, manejo de dinero, etc.), que permitan hacer frente al problema, superarlo y mantenerse en una situación de abstinencia de juego o, más dificilmente, de juego controlado.

2. Hay una clara diferencia en los tipos de juegos de elección entre los de azar disponibles en España en las mujeres respecto a los hombres.

Tabla 3.- Distribución de los juegos en jugadores patológicos de Galicia, por sexo (se presenta en \%)

\begin{tabular}{|c|c|c|}
\hline Juego & $\begin{array}{l}\text { Jugadores } \\
\text { Hombres }\end{array}$ & $\begin{array}{l}\text { patologicos } \\
\text { Mujeres }\end{array}$ \\
\hline $\begin{array}{l}\text { Cartas } \\
\text { Lotería Nacional } \\
\text { Cupón de la ONCE } \\
\text { Cuponazo } \\
\text { Lotería primitiva } \\
\text { Bonoloto } \\
\text { Primijuego } \\
\text { Quniela de fútbol } \\
\text { Quiniela hipica } \\
\text { Máquina tragaperras } \\
\text { Máquina videojuegos } \\
\text { Bíngo } \\
\text { Casino } \\
\text { Otros }\end{array}$ & $\begin{array}{l}28.6 \\
21.4 \\
32.1 \\
25.0 \\
32.1 \\
25.0 \\
0.0 \\
25.0 \\
0.0 \\
53.6 \\
25.0 \\
21.4 \\
0.0 \\
7.1\end{array}$ & $\begin{array}{l}14.3 \\
21.4 \\
25.0 \\
21.4 \\
25.0 \\
21.4 \\
10.7 \\
7.1 \\
3.6 \\
25.0 \\
7.1 \\
25.0 \\
7.1 \\
3.6\end{array}$ \\
\hline \multicolumn{3}{|c|}{$\begin{array}{l}\text { Nota: En este grupo, en su conjunto, tenia como juego } \\
\text { diario predominante las máquinas tragaperras ( } 50 \%) \text {, segui- } \\
\text { do del cupon de la ONCE ( } 25 \%) \text {, máquinas de videojuegos } \\
(21.4 \%) \text {, cartas ( } 14.3 \% \text { ) y bingo }(3.6 \%) \\
\text { Fuente: Becona (1993) }\end{array}$} \\
\hline
\end{tabular}

Es de todos sabido que los varones juegan más a las máquinas tragaperras, las mujeres a cupones y loterias y al bingo aproximadamente igual unos y otros. Esta distribución luego se vuelve a ver reflejada en los jugadores patológicos de la población o en aquellos que buscan tratamiento. No hay que olvidar que también hay otra variable moduladora: la cantidad global gastada en juego. Dado 
que son las máquinas tragaperras en donde se gasta más dinero, aquí vamos a encontrar el mayor número de jugadores patológicos de uno y otro sexo. Le siguen las loterias, aunque en este caso por tener demorado el premio, la adicción es menor que el tercer tipo de juego en importancia de recaudación, el bingo, que sí es el segundo más adictivo.

Para ejemplificar lo anterior, presentamos en la tabla 3, los datos en Galicia de los jugadores patolbgicos varones y mujeres de la población (Becona, 1993), en relación con los juegos que practican, considerando todos ellos. Alli puede apreciarse como se encuentran las diferencias anteriormente apuntadas.

3. La mujer sufre directamente cuando es jugadora patológica la doble moral o la doble visión que nuestra sociedad aplica al varón o a la mujer cuando uno $u$ otro tiene un problema o trastomo grave como es éste del juego patológico (Custer y Milt, 1985). Al varón se le tolera el juego excesivo en las primeras fases; a la mujer no. Mientras que al varón se le puede considerar una persona sin control, con poco dominio, a la mujer es más probable que se le aplique el concepto moral de "viciosa", dado que el varón todavía sigue disfrutando en nuestra sociedad de una mayor tolerancia hacia sus excesos que la mujer.

El concepto moral de "viciosa", aplicada a la jugadora o ludópata, es semejante a otros que se le han aplicado para esta misma conducta, como inmoral, indecente, descuidada, mala madre, etc. Curiosamente, estos apelativos se aplican poco o escasamente al varón con problemas de juego. La misma denominación de mujer jugadora, sin el apelativo de patológica o ludópata, ya indica una valoración negativa, o más negativa que la que se considera en el caso del hombre.

Esto en parte explica, como veremos posteriormente, que la mujer tenga más "vergüenza" que el varón en hacer público su problema, aunque lo reconoce más fácilmente que el varón para sus adentros, y acuda menos que él a los programas de tratamiento. Al acudir se siente descubierta y tiene que contar su problema. Como ocurre con el alcohol, trata de esconder su problema hasta donde puede.

4. Las fases del juego por las que pasa la mujer son las mismas que las del hombre. Estas han sido apuntadas por Custer (1984), siendo las clásicas de fase de ganancia, fase de pérdida y fase de desesperación (National Council on Problem Gambling, 1994).

Jugadores Anónimos afirma que la mitad de las mujeres jugadoras patológicas comienzan a jugar como un modo de escapar de los problemas que la abruman, incluyendo trastornos en la infancia, relaciones dificiles (como por ejemplo, esposo jugador patológico, alcohólico o con enfermedad mental), soledad y aburrimiento (ej., cuando el marido trabaja largos periodos fuera del hogar o lejos del mismo).

Les atrae la acción del juego, la activación que produce (Brown, 1988; Walker, 1992), la tensión que incrementa su humor. En este contexto el juego se convierte en un anestésico o en un hipnótico, gracias al cual se olvidan los problemas y se pasa a otro estado, de modo semejante a un estado disociativo o de cuasi-disociación (Jacobs, 1989a).

En poco tiempo, el juego como problema, abre la puerta a otros problemas, convirtiéndose el juego en el problema (monetario) y en el único modo que ven para salir de ese problema, al verse en la necesidad de ganar para recuperar las pérdidas, dados los errores cognitivos que sobre el azar son caracteristicos de los jugadores patológicos (Ladouceur, 1993).

Hay una fase final, introducida recientemente por Lesieur y Rosenthal (1991), la fase de desesperanza, en la cual la persona se ve sin salida ante su problema. A este estado llegan muchas jugadoras patológicas y en ocasiones facilita que acudan a tratamiento por no ver ya ninguna salida a su problema.

5. En la población general es bien conocido que la mujer tiene mayores problemas de depresión que el varón. Se calcula que en las sociedades industrializadas la prevalencia de depresión mayor es del 2 al $4 \%$ para los hombres y del 4 al $9 \%$ para las mujeres, que sube en el riesgo a lo largo de toda la vida entre el 7 y el $10 \%$ en hombres y del 14 al $20 \%$ en mujeres (Friedman y Thase, 1995). Como sabemos también, la depresión es la principal causa que conduce a la muerte por suicidio.

La depresión es un trastorno sumamente relevante en la mujer jugadora patológica. Sabemos que hay una asociación entre juego patológico y depresión (ej., McCormick, Russo, 
Ramirez y Taber, 1984), como ocurre igualmente en España (ej., Becoña, Lorenzo y Fuentes, 1996).

Por tanto, la relación entre depresión y juego patológico en la mujer puede ser triple: 1) es un trastorno que se da en mayor proporción que en el varón en la población general $y$, por tanto, también entre las mujeres jugadoras patológicas, y 2) en ocasiones el juego es el "escape", un nuevo término incluido en los criterios diagnósticos del DSM-IV para el juego patológico (American Psychiatric Association, 1994), el escape de los problemas cotidianos a los que no se les ve salida, y cree que a través del juego excesivo pude encontrarla; $y$, 3) el juego excesivo puede servir de activación, para así superar el estado de ánimo deprimido, a través del juego, siviendo por tanto como un modo de terapia para la depresión, auto-terapia que va a causar otros problemas aún más graves en el futuro al mantenerse o agravarse la depresión y surgir el problema del juego patológico y sin resolverse el problema de depresión.

Por nuestra experiencia, en ocasiones el juego le sirve a la persona para incrementar su humor y de ahi que le sea adaptativo en las primeras fases del mismo, pero al incrementarse la cantidad jugada y entrar en la adicción al juego se encuentra con dos problemas, el que tenía de depresión, y uno nuevo: la dependencia al juego.

Tampoco debemos olvidar que el juego patológico produce en algunos casos procesos depresivos por las consecuencias personales que a la persona le ha acarreado el juego. La probabilidad de tener este estado asociado al juego patológico va a depender de la fase de deterioro en que se encuentre el jugador.

Todo lo anterior sugiere, por tanto, que un porcentaje importante de los jugadores patológicos tiene asociados problemas de depresión. En cambio, no hay un total acuerdo si la depresión es previa o posterior al problema de juego y sobre esta cuestión se han planteado hipótesis opuestas.

6. El marido de una mujer jugadora patológica reacciona muy mal ante el juego de su mujer, al contrario de cuando es él quien tiene problemas de juego. Como afirman Custer y Milt (1985), en muchos casos la postura del marido es claramente de una "actitud punitiva irracional". Una vez más aparece aqui la diferente conceptualización social de quien padece el problema, si el varón o la mujer.

El marido, así como la familia de la jugadora patológica, no está preparada para los cambios en su conducta ya que ésta sufre grandes cambios, gasta mucho dinero, está fuera por la noche, miente a cada oportunidad y discute la mayor parte del tiempo (Lorenz, 1987).

El marido toma con gran disgusto el problema de su mujer. Para la mayoria de ellos la conducta de su mujer la hacen semejante a si ella fuese una alcohólica o una prostituta, dos de los apelativos más negativos y peyorativos que socialmente se aplican a una mujer.

Al descubrir el marido el juego en su mujer y como ha conseguido el dinero, engañandole o robándole, según su concepción, ve ahora a su mujer como una persona disoluta, inmoral y una ladrona por el dinero que le ha quitado para mantener el juego.

El marido puede pagar los gastos de su juego inicialmente, pero él es menos probable que le proporcione a ella la ayuda y comprensión que necesita desesperadamente. El marido de la jugadora patológica parece situar los límites al principio del curso del problema de ella. En este sentido la empuja a abandonar el juego rápidamente.

Sobre los hijos, el marido considera que es una mala madre, aparte de ser una mala esposa.

En muchos casos los varones no son capaces de admitir tener una mujer jugadora patológica y a lo largo del proceso de descubrimiento del problema o de la recuperación de ella piden el divorcio. También es reaccio a acudir a las sesiones de tratamiento, como ocurre igualmente en otros trastornos (ej., cuando el hijo es el que tiene un problema psicológico de cualquier tipo, habitualmente va la madre y más escasamente va el padre, o simplemente, no asiste). Este hecho incide negativamente en la mujer, de ahi que las consecuencias de su juego sean mucho más graves a nivel personal y familiar que en el varón jugador patologico.

7. Los hijos sufren de modo muy acusado las consecuencias de tener una madre jugadora patológica, como ocurre igualmente cuando lo es su padre. 
Los resultados del estudio de Jacobs (1989b) mostraron que, cuando se comparan iguales en sexo y edad de similar lugar de residencia, los hijos de jugadores problema tienen mucho mayor riesgo de tener un hogar roto, mostrar una alta incidencia en psicopatología infantil y una mayor implicación en conductas perjudiciales para la salud.

Además de sufrir las consecuencias del deterioro económico y estar presentes en las disputas de sus padres, los niños de estas familias son el blanco del abuso parental, tanto físico como verbal (Lorenz y Shuttlesworth, 1983).

Los hijos pequeños, teniendo unos pobres modelos, tanto en su padre como en su madre para enfrentarse con las dificultades de la vida, recurren a varias conductas destructivas, incluyendo ausencia sin permiso y otras formas inadecuadas de actuar. Muchos de estos niños desarrollan trastornos psicosomáticos tales como alergias, asma, problemas digestivos crónicos o dolores de cabeza (Lorenz y Yaffee, 1988).

Los hijos pequeños de jugadores patológicos creciendo en una atmósfera de deprivación emocional, aislamiento, abuso paterno, rechazo, pobres modelos de rol y énfasis en el dinero, es probable que tengan igualmente vidas con problemas. Custer y Milt (1985) resaltaron que entre los miembros de Jugadores Anónimos existen historias de juego de tres generaciones.

Estos niños tienen un perfil donde incide tanto el padre como la madre (Franklin y Thoms, 1989). Muchos niños de tales hogares tienen un importante conflicto de rol. Algunos han tomado en la familia el papel de chivo expiatorio; otros de pacificador; $y$ otros cargan de excesivas responsabilidades. A menudo, con el aumento de los sentimientos de ansiedad, ira y/o depresión, estos niños se sienten responsables del clima emocional en el hogar. Reaccionan ante este problema de varias maneras: rendimiento académico inconsistente, abuso de sustancias y conducta de juego.

Los niños de los jugadores patológicos tienen escasas habilidades sociales. Estos niños intentan conectar emocionalmente y algunas veces sienten la necesidad de negar la actividad del jugador. Muchos, al igual que los niños de cualquier padre adulto, tienen una larga historia de decepción. El padre y la madre hacen promesas que ellos nunca mantendrán y la confianza disminuye cuando el niño aprende a no creer lo que ellos escuchan. Cuando la madre se lo pide se alian con ella en contra del padre. La crisis de roles les lleva frecuentemente a imitar otros roles distintos a los de los padres (cfr., McCormick y Taber, 1987)

Estos niños a veces son muy activos físicamente: participan en deportes, juegos y cualquier tipo de tareas. Intentan ser socialmente aceptados pero sus escasas habilidades sociales no le permite hacer amigos o tienen muy pocos.

El un estudio de Jacobs (1989b), realizado con chicos de los grados 9 a 11 (equivalente al bachillerato), encontró que cuando los chicos consideraron que tenían padres con juego problemático, tenian mayores niveles de uso de alcohol, tabaco y drogas en los últimos doce meses que sus compañeros con padres sin problemas de juego. Globalmente, los jóvenes de padres jugadores mostraron una mayor preferencia por las drogas estimulantes que sus compañeros con padres sin problemas de juego.

Los chicos con padres jugadores estuvieron implicados en mayores niveles de juego y en más problemas relacionados con el juego. Para Jacobs (1989b) su mayor descubrimiento fue que el $75 \%$ de los chicos cuyos padres fueron jugadores problema informaron de una edad de aparición del juego anterior a los 11 años, comparado con el $34 \%$ de sus compañeros con padres promedio. Los chicos de jugadores problema tenian una mayor tendencia a escapar de la realidad, mayor propensión a buscar sustancias que elevasen su humor y búsqueda de experiencias estimulantes, que sus compañeros con padres promedio.

Cuando se les preguntó por su principal objetivo cuando consumian, en una lista de sustancias potencialmente adictivas, todos ellos señalaron que la estimulación o la relajación era el principal objetivo. Sin embargo, una elección alternativa fue una serie de items relacionados con el escape (de la tensión emocional, de un hogar infeliz o de una vida aburrida). Los chicos de los jugadores problema seleccionaron esta serie de items como su principal meta para consumir. 
La incidencia de indicadores de riesgo psicosocial entre estos chicos fue mucho mayor que entre sus compañeros. Así, los niños con padres jugadores experimentaron casi dos veces la incidencia de hogares rotos causados por la separación, divorcio o la muerte de uno de los padres antes de alcanzar la edad de 15 años. También indicaron mucha mayor preocupación en los chicos sobre "pensar" y "soñar sobre" distintas sustancias (ej., alcohol), así como mayor incidencia de disforia, ansiedad, depresión y riesgo de suicidio.

8. La mujer tiene un mayor control en la obtención de dinero para el juego que el varón. Esto la lleva a realizar menos actos ilegales, como robos, falsificaciones, actos violentos, etc. (Lesieur, 1993b). Esto también podria explicar la interiorización de su problema, bien a través de procesos depresivos bien a través de trastornos psicosomáticos, que son los sintomas más característicos de muchas mujeres jugadoras. De este modo su problema real, el juego, se mantiene ante los demás más latente porque solo raramente traspasa los límites sociales como son el robo, empenar todo, falsificar, engañar a familiares o conocidos, etc.

La mujer jugadora suele ser ama de casa - trabaja en profesiones donde gana menos dinero que el varón (ej., Becoña, 1993). De ahi que sus deudas, tanto en uno como en otro caso, sean menores y se vea menos abocada a conseguir dinero de modo ilegal. Acuden más a la obtención del dinero en la familia, a diferencia del hombre que lo hace más fuera de ella. Ello facilita una contención de la espiral de deterioro.

Tener hijos puede ser otro importante elemento de contención para no llegar a la espiral de total deterioro o desesperanza. Es como si aceptase los valores sociales imperantes sobre of rol tradicional de mujer, lo que le facilitaria no transgredir ciertos límites, después de los cuales es difícil la marcha atrás.

Un estudio relevante en este sentido es el de Lesieur (1993a), donde analizaron los delitos cometidos por mujeres jugadoras patológicas, utilizando para ello dos muestras: una de 50 mujeres jugadoras patológicas que asistian a capitulos de Jugadores Anónimos (JA), y otra de 114 mujeres prisioneras en Nueva Jersey, de las cuales el $34 \%$ eran jugadoras patológicas.
Entre unas y otras había semejanzas $y$ diferencias interesantes. Así, eran también alcohólicas el $14 \%$ de las que acudian a JA y un $36 \%$ las que estaban en la cárcel; también dependian de las drogas el $22 \%$ de las que asistian a JA y un $59 \%$ de las que estaban en la cárcel. Con los criterios de abuso de drogas del DSM-III-R, un $56 \%$ de las mujeres jugadoras patológicas que asistian a JA y un $88 \%$ de las que estaban en la cárcel tenian el diagnóstico de abuso de drogas en algún punto de su vida. Lo anterior indica un nivel doble o triple en estos trastornos que lo que se encuentra en la población adulta femenina norteamericana.

Respecto a sus padres, las mujeres que acudian a JA, indicaron que un $28 \%$ de sus padres era alcohólico, un $10 \%$ de sus madres alcohólicas, un $20 \%$ de sus padres jugadores compulsivos, un $4 \%$ de sus madres jugadoras compulsivas y un $12 \%$ de ellos tenian otros graves problemas. Entre las jugadoras patológicas casadas, el $62 \%$ lo estaba con un marido que era jugador patológico, alcohólico, dependiente de drogas, tenia otras adiciones o problemas mentales. Entre éstos un $19 \%$ eran jugadores patológicos y un $32 \%$ alcohólicos. Como caracteristica de ello, un factor común en casi todas las mujeres jugadoras patológicas de esta muestra es la ausencia del marido de casa. La mitad de estas mujeres afirman que comenzaron a jugar como un modo de escape de sus problemas agobiantes, como trauma, relaciones maritales problemáticas y la soledad.

El dinero para jugar lo obtienen fundamentalmente del dinero de la casa, propiedades personales, créditos bancarios, amigos $e$ incluso de cogérselo al marido y a los hijos. Un cuadro semejante se aprecia en los jugadores paíológicos en la cárcel.

Una de las conclusiones más interesantes de este estudio es que las mujeres jugadoras patológicas que acudian a JA era más probable que utilizasen procedimientos legales para obtener dinero para su juego que lo que ocurría cuando eran jugadoras patológicas encarceladas. La explicación de esta discrepancia parece estar en una variable moduladora: la adicción al alcohol o drogas. Cuando la mujer tiene dependencia del alcohol o drogas tiene como problema añadido el cometer actos ilega- 
les, sea o no jugadora patológica. Esto apareció reflejado en este estudio en ambas muestras.

9. La mujer comienza a jugar más tarde que el varón. En muchos casos su conducta de juego problemático aparece en una etapa media o tardia de la vida, hecho claramente diferencial al del varón.

Hoy sabemos, por los escasos datos que tenemos en los jóvenes en nuestro pais (Becoña y Gestal, 1995; Villa, Becoña y Vázquez, 1996) que la relación entre varones y mujeres jugadores patológicos es claramente favorable al varón en edades tempranas, entre 11 y 16 años, con una relación de 9 a 1. Este hecho se debe a que el varón comienza a jugar antes que la mujer, como igualmente hace en otras conductas como fumar, beber alcohol, etc. (Mendoza, Sagrera y Batista, 1994).

La mujer, incluso, puede no llegar a considerar el juego como algo relevante. Sin embargo, puede llegar a descubrirlo en una etapa posterior de su vida. Este fenómeno de la progresión caracteriza a muchas mujeres, especialmente a aquellas que comienzan a jugar a los 30 o 40 años. Algunos de los ejemplos ya expuestos anteriormente iban en esta linea.

10. No es extraño encontrar un elevado nivel de psicopatología en las mujeres que acuden a tratamiento, asi como una historia pasada llena de rechazos, indiferencia y traumas (Custer y Milt, 1985). Por lo ya visto, las jugadoras patológicas que acuden a tratamiento -las pocas que acuden a tratamiento-, son las más deterioradas. De ahí que la visión clínica que se posee de ellas no se adecúa a la realidad de todas las mujeres jugadoras patológicas, especialmente las que no acuden a tratamiento.

Esto parece confirmarse en los estudios epidemiológicos. En éstos la relación mujerhombre es claramente favorable al varón (ej., Becoña, 1993; Becoña y Fuentes, 1995; Irurita, 1996), pero claramente distinta a la relación que luego nos encontramos con los varones que acuden a tratamiento respecto a las mujeres, en donde están claramente sobrerepresentados en relación a los que existen en la población general.
Algunas, cuando acuden a tratamiento lo hacen en un estado penoso: las ha abandonado su marido, han perdido a sus hijos, su familia y amigos se desentiende de ella, por lo que se encuentran desesperadas. De ahi, la importancia de concienciar no solo a las mujeres con problemas de juego de que acudan a tratamiento, sino a todo el conjunto de la sociedad para que asuma que estamos hablando de un problema grave y que las personas que lo padecen precisan en muchos casos nuestra ayuda, no la económica, sino el apoyo que habitualmente se les suele negar.

Strachan y Custer (1993) han analizado las caracteristicas de las mujeres jugadoras patológicas que asistian a las reuniones de Jugadores Anónimos en Las Vegas. De éstas, el $76 \%$ estaban en la década de los 30 o 40 años, el $67 \%$ casadas y el $75 \%$ tenian niños.

En el $31 \%$ de los casos los padres se habian divorciado antes de ellas cumplir los 15 años. Un $31 \%$ habian perdido al esposo, padre, hijos o amigos cercanos, por muerte, en los dos años previos a asistir a Jugadores Anónimos. El $42 \%$ tenía un padre alcohólico y el $42 \%$ tenian un padre o padres que jugaban mucho. El $29 \%$ afirmaba que habia sufrido abusos sexuales de niña. A nivel de salud mental, el $69 \%$ se habian planteado el suicidio y el $23 \%$ lo habia intentado. El $10 \%$ habia abusado del alcohol en el pasado y un $\mathbf{2 3} \%$ de las drogas, junto a un $15 \%$ de fármacos.

El $90 \%$ de ellas eran adictas a las máquinas tragaperras (video poker machines). También el $90 \%$ afirmaban que cuando jugaban lo hacia ininterrumpidamente por periodos que oscilaban entre 7 y 25 o más horas consecutivas. Ello es posible hacerlo en Las Vegas (cfr. Becoña, 1996), a diferencia de nuestro medio.

El nivel de deterioro de ellas era evidente: el $73 \%$ obtenian dinero para jugar falsificando cheques, el $87 \%$ gastando los ahorros familiares y el $76 \%$ gastando el crédito de sus tarjetas de créditos. El $60 \%$ vendió o empeñó las joyas, otras habian pedido adelantos de dinero en el trabajo, etc. Estos índices son un claro indicio de deterioro (American Psychiatric Association, 1994).

Un $27 \%$ de estas mujeres habian considerado prostituirse para pagar su juego y un $10 \%$ lo hacian en el momento de realizar el estudio, cuando estaban en tratamiento. 
11. Como ya hemos visto, se encuentran antecedentes en muchos casos, tanto en los jugadores patológicos varones como mujeres, de padres o madres jugadores patológicos 0 padres con problemas de abuso o dependencia del alcohol.

El alcohol y el juego son malos amigos para el jugador patológico. Algo semejante ocurre con el alcohol y el juego de sus progrenitores. Cuando asl ocurre éste es un claro predictor negativo del juego patológico, su aparición, desarrollo y recuperación. Un amplio conjunto de estudios han analizado esta relación (ver Becona, Fuentes y Lorenzo, 1993).

Tabla 4.- Consumo de distintas sustancias adictivas en jugadores patológicos varones y mujeres en Galicia

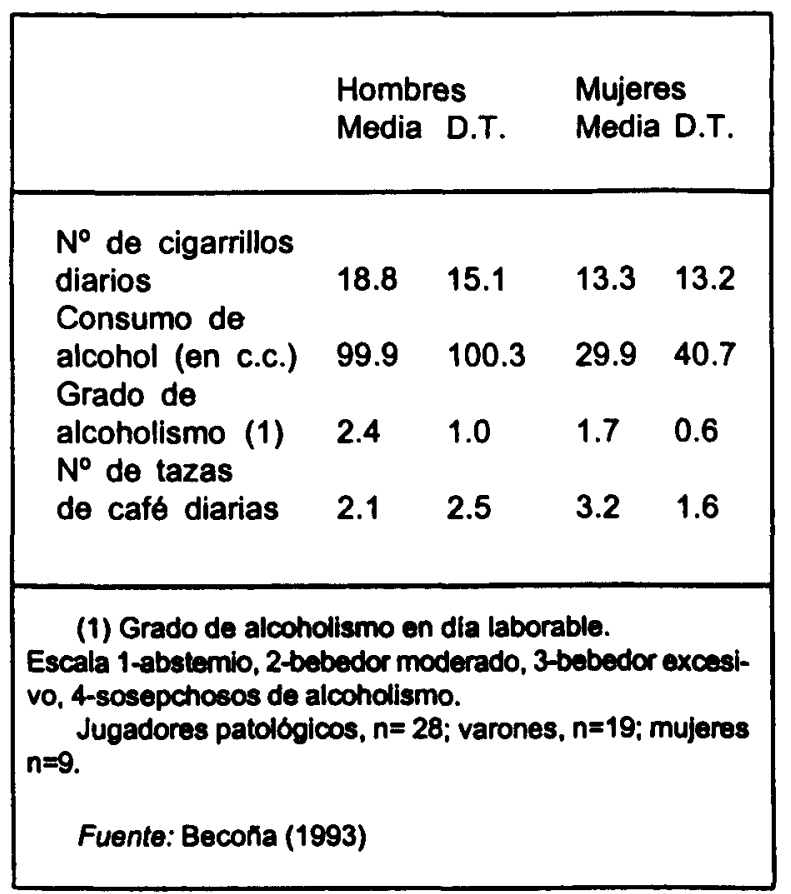

Como deciamos al principio, hay en ocasiones grandes paralelismos entre la mujer alcohólica y la mujer ludópata en su comportamiento. Uno de estos es que cuando tiene dependencia del alcohol muchas de ellas beben a escondidas, en sus casas, no de modo público en los bares como los varones. Algo parecido encontramos en algunas mujeres dependientes del juego. Especialmente relevante es esto en la jugadora patológica de cupones y lote- rias. Juega de una forma socialmente bien vista, nadie aprecia que puede tener un problema de dependencia al juego, y sus problemas los llora en casa, tanto de pérdida de dinero, como los trastornos psicosomáticos, la depresión o la búsqueda imperiosa de dinero para seguir jugando, que pueden estar asociados al juego.

En el estudio de Lesieur (1988), con mujeres jugadoras patológicas, el $50 \%$ de ellas habia abusado del alcohol, drogas o ambas en algún momento de su vida. El $24 \%$ también se consideraban gastadoras compulsivas, el $20 \%$ comian compulsivamente y el $12 \%$ con una posible adicción al sexo.

Baja autoestima, sentimientos de inutilidad, una carga de dolor indescriptible y de sentirse abandonada por todos, son algunas de las caracteristicas que podemos observar en ellas.

12. El tratamiento. En Estados Unidos solo acuden a las sesiones de tratamiento de Jugadores Anónimos entre un 2 y un $4 \%$ de mujeres (Lesieur, 1990). Ello lo achacan al estigma que tiene la mujer jugadora patológica. Casi todas las que acuden son mujeres casadas, acuden voluntariamente por ellas mismas, como encontró Lesieur (1988) en su estudio, donde asi lo hacia el $62 \%$. A diferencia de los varones, la mitad de las mujeres que acuden a la primera sesión no volverán a otra (Lesieur, 1988).

La situación en España es semejante: acuden entre el 10 y el $20 \%$ del total de los jugadores que están en tratamiento (Echeburúa et al., 1994; Sáiz et al., 1992). La evidencia indica que el número de mujeres jugadoras patológicas en la población es mayor, pero acuden menos a tratamiento, fenómeno que ocurre en todos los paises (ej., Lesieur, 1993b; Walker, 1992).

Cuando una mujer jugadora patológica acude a tratamiento lo hace sumisa, reservada, asustada, abatida y retraída en su comportamiento (Custer y Milt, 1985). Cuando es varón, éste aparece como más egoista y orgulloso y no admite su trastorno, a diferencia de la mujer. Esta suele reconocerlo. Cuando no lo hace es porque se siente avergonzada de reconocerlo y por las implicaciones que le tiene, que son mucho peores que en el caso del varón. 
Custer y Milt (1985) resumen las diferencias entre el jugador patológico varón y mujer, indicando que cuando acuden a tratamiento los varones son dificiles, egoistas, obstinados, punitivos, sin sentimientos, incapaces de empatizar. Por contra, las mujeres son sumisas, con un evidente sufrimiento, y están preocupadas, pero se muestran afectuosas $y$ empáticas.

Aunque Custer y Milt (1985) se plantean la cuestión de si lo anterior es biológico o cultural. Claramente habria que hablar de una síntesis de ambos aspectos de la persona: la mujer tiene unas características personales moduladas por la biología, al tiempo que la cultura se encarga de potenciarle algunos aspectos biológicos o permitir surgir otros que solo la cultura modula.

En Estados Unidos tiene una gran relevancia para el tratamiento del juego patoIógico Jugadores Anónimos. A partir de Jugadores Anónimos (Gamblers Anonymous) ha surgido Gam-Anon y Gam-a-Teen. La primera está orientada a las mujeres de los jugadores y la segunda a los hijos de ellos. Mientras que Gam-Anon va bien, el exito de Gam-a-Teen, la dirigida a los hijos, tiene escaso éxito. Estos tienen grandes problemas para afrontar la situación y los problemas que surgen a partir de la situación del padre o madre.

Cuando el juego patológico es más que un problema individual, un problema familiar, el terapeuta tiene que aplicar junto a la terapia individual y/o grupal, terapia familiar, o terapia para la familia adicta, como ocurre más frecuentemente de lo que parece (Lesieur, 1990). Asi, en Estados Unidos, el $25 \%$ de los jugadores patológicos son hijos de alcohólicos $y$ otro $20 \%$ son hijos de jugadores patológicos (cfr. Lesieur, 1993b). En este caso el tratamiento es más complejo, más difícil y con un resultado en el abordaje poco efectivo

Como ya hemos apuntado anteriormente, el marido es reaccio a acudir a las sesiones de tratamiento, bien acompañando a su mujer, bien para que conozca en qué consiste el problema de su esposa y como puede él ayudarle. Ella tiene en la mayoría de las ocasiones que sufrir esta soledad, soledad afectiva y soledad existencial en muchas ocasiones. Este hecho incide negativamente en la mujer, y en la recuperación de su pro- blema de dependencia del juego. Por tanto, la recuperación no siempre es fácil y el apoyo a veces es escaso en su medio más cercano y más querido.

En el tratamiento, hay que cubrir toda una serie de áreas claves, orientadas a resolver los problemas de la mujer jugadora, tanto relacionadas con el juego como otros. Junto al problema de juego, deben tenerse en cuenta si hay abuso de otras sustancias, especialmente alcohol, depresión asociada, trastornos psicosomáticos, problemas maritales y manejo de sus hijos, trabajo, deudas, etc.

\section{CONCLUSIÓN}

La mujer jugadora existe y tiene unas caracteristicas distintas a las del varón, aunque su problema de juego le produce los mismos problemas que en el varón. Sin embargo su historia de juego es distinta al varón, participa en juegos de azar distintos y sufre más intensamente su trastorno. Como dice el National Council on Problem Gambling (1994) norteamericano "las mujeres jugadoras compulsivas son a menudo víctimas de los estereotipos sociales y de las limitaciones impuestas sobre la población femenina". Esta estigmatización es real y la hay que tener en cuenta para ayudar a las que no quieren ayuda como a las que acuden a tratamiento.

La detección de estas mujeres con problemas graves de juego es un objetivo y una necesidad para los próximos años. La siempre hablada prevención tiene aquí todo su sentido. La pareja de la jugadora puede detectar este problema. Debe hacerlo y debe estar alerta, como si ocurre el caso contrario. Si hay indicios de la existencia del mismo, debe analizar si existe y detectarlo para buscarle una salida; negarlo no es la solución.

En la estimación de Dunne (1985), se calcula que por cada jugador patológico que existe se ven afectadas otras 10 a 20 personas entre allegados, amigos y compañeros de trabajo. Este hecho agrava el problema del jugador patológico. Así, pasar de ser no solo un problema individual, sino también social. Social porque afecta a las personas del entorno del jugador, de la mujer jugadora en este caso. Los hijos, quizás, son los más afectados, después de la propia jugadora. Sufrirán 
la falta de afecto, los problemas de carestía económica, malos tratos en ocasiones, inconsistencia en la educación, etc. Ello facilita que el nivel de psicopatología de ellos sea mayor en este periodo de su vida y en su vida adulta; muchos aprenderán en este momento a ser jugadores patológicos en su vida adulta o no tan adulta, ya en la adolescencia.

Conocer bien a la mujer jugadora patológica en particular y el trastorno del juego patológico en general es el modo de conocer la incidencia, detectar a estas personas con graves problemas y poder prestarles nuestra ayuda.

\section{REFERENCIAS BIBLIOGRÁFICAS}

American Psychiatric Association (1994). Diagnostic and statistical manual of mental disorders, revised 4th ed. Washington, D.C.: American Psychiatric Association.

Becoña, E. (1993). El juego compulsivo en la comunidad autónoma gallega. Santiago de Compostela: Consellería de Sanidade da Xunta de Galicia.

Becoña, E. (1995a). Juego patológico. En V. Caballo (coord.), Manual de psicopatologia y trastornos psiquiátricos. Vol. 1. Fundamentos conceptuales; trastornos por ansiedad, afectivos $y$ psicóticos (pp. 551-582). Madrid: Siglo XXI.

Becoña, E. (1995b). The problem and pathological gambling in Europe: The cases of Germany, Holland and Spain. Joumal of Gambling Studies, 12, 179-192.

Becoña, E. (1996). La ludopatía. Madrid: Aguilar.

Becoña, E. y Fuentes, M.J. (1995). El juego patológico en Galicia evaluado con el South Oaks Gambling Screen. Adicciones, 7, 423-440

Becoña, E., Fuentes, M.J. y Lorenzo, M.C. (1993). Guia bibliográfica sobre juego patológico. Psicología Conductual, 1, 455-468

Becoña, E. y Gestal, C. (1994). El juego patológico en niños del $2^{\circ}$ ciclo de E.G.B. de La Coruña. Psicothema, 8, 13-23.

Becoña, E., Labrador, F.J., Echeburúa, E., Ochoa, E. y Vallejo, M.A. (1995). Slot gambling in Spain: $A$ new and important social problem. Journal of Gambling Studies, 11, 265-286.

Becoña, E., Lorenzo, M.C. y Fuentes, M.J. (1996). Pathological gambling and depression. Psychological Reports, 78, 635-640.
Brown, R.I.F. (1988). Reversal theory and subjective experience in the explanation of addiction and relapse. En M.J. Apter, J.H. Kerr y M.P. Cowles (Eds.), Progress in reversal theory (pp. 191211). North, Holland: Elsevier Science Publishers.

Cayuela, R. (1990). Characteristics and situation of gambling addiction in spain: Epidemiological and clinical aspects. Paper presented at the Eight International Conference on Risk and Gambling, London, August.

Comisión Nacional del Juego (1996). Memoria del Juego de 1995. Madrid: Ministerio del Interior, Comisión Nacional del Juego.

Custer, R.L. (1984). Profile of the pathological gambler. Joumal of Clinical Psychiatry, 45, 35-38.

Custer, R. y Milt, H. (1985). When luck runs out. Help for compulsive gamblers and their families. Now York: Facts on File Publications.

Dunne, J.A. (1985). Increasing public awareness of pathological gambling behavior. Journal of Gambling Studies, 1, 8-17.

Echeburúa, E. (1996). El alcoholismo. Madrid: Aguilar.

Echeburúa, E., Báez, C. y Fernández-Montalvo, J. (1996). Comparative effectiveness of three therapeutic modalities in the psychological treatment of pathological gambling: Long-term outcome. Behavioural and Cognitive Psychotherapy, 24, 51-72.

Edwards, G. (1986). Tratamiento de alcohólicos. Guia para el ayudante profesional. México: Trillas.

Fleming, A.M. (1978). Something for nothing: A history of gambling. New York, NY: Delacorte Press.

Franklin, J. y Thoms, D.R. (1989). Clinical observations of family members of compulsive gamblers. En: H.J. Shaffer, S.A. Stein, B. Gambino y T.N. Cummings (Eds.), Compu/sive gambling. Theory, research, and practice (pp. 135-146). Lexington, MA: Lexington Books.

Friedman, E.S. y Thase, M.E. (1995). Trastomos del estado de ánimo. En Caballo, V. (Coord.), Manual de psicopatología y trastomos psiquiátricos. Vol. 1. Fundamentos conceptuales; trastornos por ansiedad, afectivos y psicóticos (pp. 619-681). Madrid: Siglo XXI.

González, A. (1989). Juego patologico: una nueva adicción. Madrid: Canal Comunicciones.

Irurita, I.M. (1996). Estudio sobre la prevalencia de los jugadores de azar en Andalucia. Sevilla: Comisionado para la Droga, Consejería de Asuntos Sociales, Junta de Andalucia.

Heineman, M. (1992). Losing your shirt. Recovery for compulsive gamblers and their families. Minneapolis, Ml: CompCare Publications. 
Jacobs, D.F. (1989a). A general theory of addictions: Rationale for and evidence supporting a new approach for understanding and treating addictive behaviors. En H.J. Shaffer; S.A. Stein; B. Gambino, y T.N. Cummings (Eds.), Compulsive gambling. Theory, research, and practice (pp. 35-64). Lexington, MA: Lexington Books.

Jacobs, D.F. (1989b). Illegal and undocumented: A review of teenage gambling and the plight of children of problem gamblers in America. En H.J. Shaffer; S.A. Stein; B. Gambino, y T.N. Cummings (Eds.), Compulsive gambling. Theory, research, and practice (pp. 249-292). Lexington, MA: Lexington Books.

Labrador, F.J. y Becoña, E. (1994). Juego patológico: Aspectos epidemiológicos y teorías explicativas. En J.L. Graña (ed.), Conductas adictivas: Teoria, evaluación y tratamiento (pp. 495-520). Madrid: Debate.

Ladouceur, R. (1993). Aspectos fundamentales y clínicos de la psicologia de los juegos de azar. Psicologia Conductual, 1, 361-374.

Legarda, J.J., Babio, R. y Abreu, J.M. (1992). Prevalence estimates of pathological gambling in Seville (Spain). British Joumal of Addictions, 87, 767-770.

Lesieur, H.R. (1988). The female pathological gambler. En W.R. Eadington (Ed.), Gambling Studies: Proceedings of the 7th International Conference on Gambling and Risk Taking. Reno, $\mathrm{NJ}$ : University of Nevada.

Lesieur, H. (1990). Working with and understanding Gamblers Anonymous. En T.J. Powell (ed.), Working with self-help (pp. 237-253). Silver Spring, MD: NASW Press.

Lesieur, H.R. (1993a). Female pathological gamblers and crime. En W.R. Eadington y J.A. Cornelius (eds.), Gambling behavior \& problem gambling (pp. 495-515). Reno, NV: Institute for the Study of Gambling and Commercial Gaming.

Lesieur, H.R. (1993b). Prevalencia, caracteristicas y tratamiento de los jugadores patológicos en Estados Unidos. Psicologia Conductual, 1, 389407.

Lesieur, H.R. y Rosenthal, R.J. (1991). Pathological gambling: $A$ review of the literature (Prepared for the American Psychiatric Association Task Forece on DSM-IV Committee on Disorders of Impulse Control Not Elsewhere Classified). Journal of Gambling Studies, 7, 5-39.

Lorenz, V.C. (1987). Famimy dynamics of pathological gamblers. En T. Galski (ed.), The handbook of pathological gambling (pp. 71-88). Springfield, IL: Charles C. Thomas Publisher.
Lorenz, V.C. y Shuttlesworth, D.E. (1983). The impact of pathological gambling on the spouse of the gambler. Journal of Community Psychology, 11, 67-76.

Lorenz, V.C. y Yaffee, R.A. (1988). Pathological gambling: Psychosomatic, emotional and marital dificulties as reported by the spouse. Joumal of Gambling Behavior, 4, 13-26.

McCormick, R.A., Russo, A.M., Ramirez, L.F. y Taber, J.I. (1984). Affective disorders among pathological gamblers in treatment. American Journal of Psychiatry, 141, 215-218

McCormick, R.A. y Taber, J.I. (1987). The patological gamblers: Salient personality variables. En: T. Galski (Ed.), The handbook of pathological gambling (pp. 9-39). Springfields, IL: Charles C. Thomas Publisher.

Mendoza, R., Sagrera, M.R. y Batista, J.M. (1994). Conductas de los escolares españoles relacionadas con la salud (1986-1990). Madrid: Consejo Superior de Investigaciones Cientificas

National Council on Problem Gambling (1994). Women who gamble too much. New York, NY: National Council on Problem Gambling.

Ochoa, E. y Labrador, F.J. (con la colaboración de E. Echeburia, E. Becona y M.A. Vallejo) (1994). El juego patológico. Barcelona: Plaza \& Janés.

O.M.S. (1992). CIE-10. Décima revisión de la clasificación internacional de las enfermedades. Trastornos mentales y del comportamiento. Descripciones clinicas y pautas para el diagnóstico. Madrid: Meditor.

Robert, C. y Botella, C. (1995). Trastornos de control de impulsos: el juego patológico. En A. Belloch, B. Sandín y F. Ramos (Eds.), Manual de psicopatologia, vol. 1 (pp. 559-594). Madrid: McGraw-Hill.

Sáiz, J., Moreno, I. y López-Ibor, J.J. (1992). Ludopatia: estudio clínico y terapéutico-evolutivo de jugadores patológicos. Actas Luso-Españolas de Neurología y Psiquiatría, 20, 189197.

Strachan, M.L. y Custer, R.L. (1993). Female compulsive gamblers in Las Vegas. En W.R. Eadington y J.A. Cornelius (Eds.), Gambling behavior \& problem gambling (pp. 235-238). Reno, NV: Institute of the Study of Gambling and Commercial Gaming, University of Nevada, Reno.

Villa, A., Becoña, E. y Vázquez, F.L. (1996). El juego patológico en niños $y$ adolescentes de Asturias. Manuscrito pendiente de publicación.

Walker, M.B. (1992). The psychology of gambling. Oxford, R.U.: Pergamon Press. 